International Journal of Pure and Applied Mathematics

Volume 84 No. 4 2013, 321-329

ISSN: 1311-8080 (printed version); ISSN: 1314-3395 (on-line version)

url: http://www.ijpam.eu

doi: http://dx.doi.org/10.12732/ijpam.v84i4.2

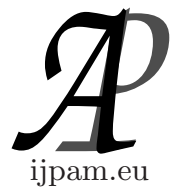

\title{
A NOVEL APPROACH FOR A CLASS OF HIGHER ORDER NONLINEAR SINGULAR BOUNDARY VALUE PROBLEMS
}

\author{
K. Aruna ${ }^{1 \S}$, A.S.V. Ravi Kanth ${ }^{2}$ \\ Fluid Dynamics Division \\ School of Advanced Sciences \\ V.I.T. University, Vellore, 632 014, Tamil Nadu, INDIA
}

\begin{abstract}
In this paper, we present a novel approach for solving higher order nonlinear singular boundary value problems (SBVP). The original differential equation is modified at the singular point then the boundary value problem is treated by differential transform method(DTM). The proposed method is a promising tool for solving a class of higher order nonlinear singular boundary value problems and is capable of reducing the computational size. Numerical examples are included to demonstrate the validity and applicability of the technique and a comparison is made with the existing results.
\end{abstract}

AMS Subject Classification: 65L10, 34B16

Key Words: singular nonlinear boundary value problems, differential transform method, differential equations, series solutions

\section{Introduction}

The aim of this paper is to introduce a novel approach for solving a class of higher order nonlinear singular boundary value problems:

$$
y^{(m)}(x)+\frac{\alpha}{x} y^{(m-1)}(x)=f(x, y)
$$

subject to the boundary conditions

Received: August 28, 2012

(c) 2013 Academic Publications, Ltd.

$\S$ Correspondence author url: www.acadpubl.eu 


$$
y(0)=\gamma_{0}, \quad y^{\prime}(0)=\gamma_{1}, \ldots, \quad y^{(m-1)}(0)=\gamma_{m-1}, \quad y(1)=\mu \text { or } y^{\prime}(1)=\omega
$$

These type of problem occurs frequently in various areas of engineering and scientific applications for example in the study of electro hydrodynamics, theory of thermal explosions, reactant concentration in a chemical reactor, boundary layer theory, and flow networks in biology, the study of astrophysics, the deflection of a curved beam having a constant or varying cross section, three layer beam, electromagnetic waves or gravity driven flows, beam theory, inelastic flows, the theory of elastic stability and so on. Finding accurate and efficient methods for solving higher order SBVP's has been an active research undertaking. Numerical treatment for higher order SBVP's are challenging because of singularity behavior at the origin. Sharma and Gupta [1] studied the multi-integral method for solving non singular fourth order differential equations. Yousef et.al [2], applied an integral method to solve singular fourth-order boundary value problems. In [3] the original differential equation is modified at singular point then the boundary value problem is treated by using cubic splines. DTM for solving singular boundary value problems was studied in [4]. The authors of [5-6] applied modified Adomain decomposition method to solve higher order SBVP's. The numerical study of SBVP's has been given in [7-12] and references therein. To the authors knowledge no paper has been reported yet for the solution of higher order nonlinear SBVP's using DTM. In this paper, we introduced the DTM as an alternative to existing methods for solving higher order nonlinear SBVP's. The use of DTM in electric circuit analysis was pioneered by Zhou [13] and later adopted by several researchers [14-22]. The DTM obtains an analytical solution in the form of a polynomial. It is different from the traditional high order Taylor's series method, which requires symbolic competition of the necessary derivatives of the data functions. The Taylor series method is computationally taken long time for large orders. With this method, it is possible to obtain highly accurate results or exact solutions for differential equations. The paper is organized in four sections. Basic concept of DTM is discussed in Section 2. The present method has been applied to the higher order singular boundary value problems in Section 3. In Section 4 the method is applied to three test problems and numerical experiments are given to demonstrate the validity and the efficiency of the proposed method. 


\section{Basic Ideas of Differential Transform Method}

An arbitrary function $y(x)$ can be expanded in Taylor series about a point $x=$ 0 as

$$
y(x)=\sum_{k=0}^{\infty} \frac{x^{k}}{k !}\left|\frac{d^{k} y(x)}{d x^{k}}\right|_{x=0}
$$

The differential transform of $y(x)$ is defined [13] as

$$
Y(k)=\frac{1}{k !}\left|\frac{d^{k} y(x)}{d x^{k}}\right|_{x=0}
$$

where $y(x)$ is the original function and $Y(k)$ is the transformed function.

The differential inverse transform is

$$
y(x)=\sum_{k=0}^{\infty} Y(k) x^{k}
$$

In actual applications, the function $y(x)$ is expressed by a finite series with a remainder term and equation (5) can be rewritten as follows

$$
y(x)=\sum_{k=0}^{n} Y(k) x^{k}+R_{n}(x)
$$

which means that $R_{n}(x)=\sum_{k=n+1}^{\infty} Y(k) x^{k}$ is small, negligibly. Usually the value of $n$ is decided by convergence of the series coefficients. The fundamental mathematical operations performed by differential transform method are listed in Table 1.

\section{Description of the Method}

We first modify the Eq. (1) at the singular point at $x=0$ by using L' Hospital rule, the boundary value problem (1) is transformed into

$$
\begin{gathered}
(1+\alpha) y^{m}(0)=f(0, y(0)) \\
y^{(m)}(x)+\frac{\alpha}{x} y^{(m-1)}(x)=f(x, y), x \neq 0
\end{gathered}
$$

The differential transformed version of Eq.(7) -(8) are

$$
(1+\alpha) 1.2 .3 \ldots m Y(m)=F(0, Y(0))
$$




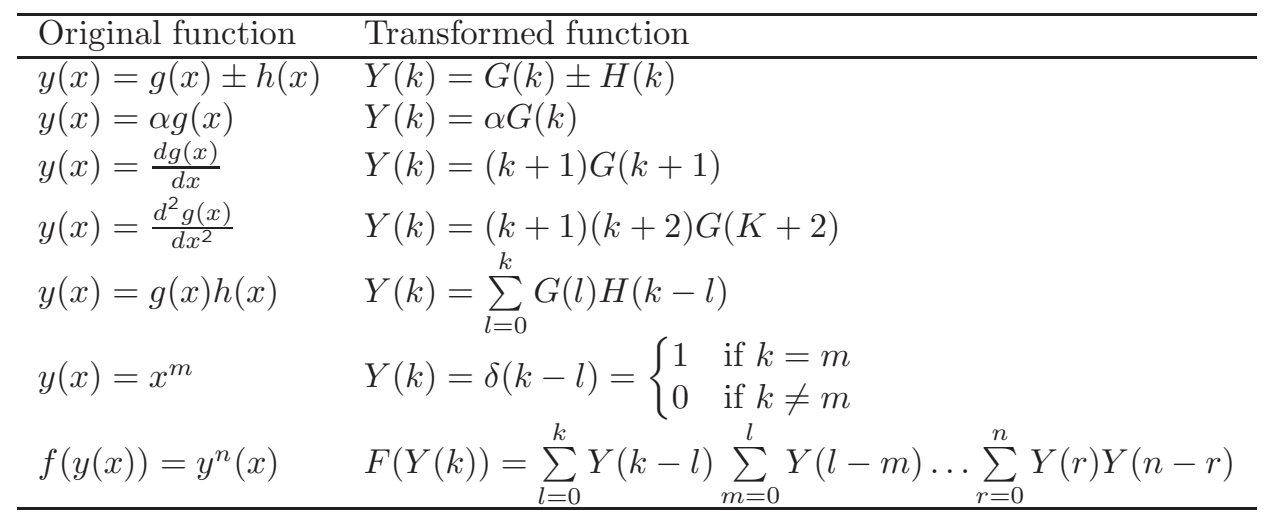

Table 1: The fundamental operations of differential transform method

$$
\begin{array}{r}
\sum_{l=0}^{k} \delta(l-1)(k-l+1)(k-l+2) \ldots(k-l+m) Y(k-l+m) \\
+\alpha(k+1)(k+2) \ldots(k+m-1) Y(k+m-1)=\sum_{l=0}^{k} \delta(l-1) F(k-l)
\end{array}
$$

where $F(k)$ is the transformed version of nonlinear function. The transformed conditions are

$$
\begin{aligned}
Y(0) & =\gamma_{0}, \quad Y(1)=\gamma_{1}, \ldots, \quad Y^{(n-1)}(0)=\frac{\gamma_{n-1}}{1.2 .3 \ldots(n-1)}, \\
\sum_{k=0}^{n} Y(k) & =\mu \quad \text { or } \quad \sum_{k=0}^{n}(k+1) Y(k+1)=\omega
\end{aligned}
$$

Substituting (11) in (10) and by recursive method we can calculate all values of $Y(k)$. Hence substituting $Y(k)$ 's in (6) we get the series solution.

\section{Numerical Results}

In this section, we tested the proposed approach for a class of higher order nonlinear singular boundary value problems.

Example 1. First, we consider the singular nonlinear third order boundary value problem

$$
y^{\prime \prime \prime}(x)-\frac{2}{x} y^{\prime \prime}(x)=y(x)+y^{2}(x)+7 x^{2} e^{x}+6 x e^{x}-6 e^{x}-x^{6} e^{2 x}
$$




\begin{tabular}{llll}
\hline$x$ & Solution in $[6]$ & DTM solution & Exact solution \\
\hline 0 & 0 & 0 & 0 \\
0.1 & 0.00110498291759 & 0.00110517367202 & 0.00110517091807 \\
0.2 & 0.00976821400349 & 0.00977126613035 & 0.00977122206528 \\
0.3 & 0.03643095870463 & 0.03644641087533 & 0.03644618780455 \\
0.4 & 0.09542864355471 & 0.09547748493395 & 0.09547678064904 \\
0.6 & 0.39333377246903 & 0.39358114034854 & 0.39357766088435 \\
0.7 & 0.69026481777657 & 0.69072328032408 & 0.69071717866237 \\
0.8 & 1.13870338730698 & 1.13948595614931 & 1.13947695538814 \\
0.9 & 1.79180568789922 & 1.79306023923106 & 1.79305066803341 \\
1.0 & 2.71636750366666 & 2.718281828459043 & 2.7182818284591 \\
\hline
\end{tabular}

Table 2: Numerical Solution for Example 1

subject to the boundary conditions

$$
y(0)=y^{\prime}(0)=0, y^{\prime}(1)=e
$$

The exact solution of this problem is $y(x)=x^{3} e^{x}$. Table 2 exhibits the errors obtained by the present method, solution in [6] and the exact solution.

Example 2. Next, we consider the singular nonlinear third order boundary value problem

$$
y^{\prime \prime \prime}(x)-\frac{2}{x} y^{\prime \prime}(x)-y^{3}(x)=7 x^{2} e^{x}+6 x e^{x}-6 e^{x}-x^{9} e^{3 x}+x^{3} e^{x}
$$

subject to the boundary conditions

$$
y(0)=y^{\prime}(0)=0, y^{\prime}(1)=10.873
$$

The exact solution of this problem is $y(x)=x^{3} e^{x}$.Comparison between the solution obtained by using the proposed method, method in [7] and the exact solution are presented in Table 3 .

Example 3. Next, we consider the singular nonlinear fourth order boundary value problem

$$
y^{i v}(x)+\frac{4}{x} y^{\prime \prime \prime}(x)=15 y^{5}(x)\left(3-7 x^{2} y^{2}(x)\right)\left(1-x^{2} y^{2}(x)\right)
$$

subject to the boundary conditions

$$
y(0)=\frac{1}{2}, y^{\prime}(0)=0, y(1)=\frac{1}{\sqrt{5}}, y^{\prime}(1)=-\frac{1}{5 \sqrt{5}}
$$




\begin{tabular}{llll}
\hline$x$ & Solution in $[7]$ & DTM solution & Exact solution \\
\hline 0 & 0 & 0 & 0 \\
0.1 & 0.00110272861806 & 0.00110498291759 & 0.00110517091807 \\
0.2 & 0.009732145252064 & 0.00976821400349 & 0.00977122206528 \\
0.3 & 0.036248360725432 & 0.03643095870463 & 0.03644618780455 \\
0.4 & 0.094851537726356 & 0.09542864355471 & 0.09547678064904 \\
0.5 & 0.204563587110123 & 0.20597260702506 & 0.20609015883752 \\
0.6 & 0.390411591946278 & 0.39333377246903 & 0.39357766088435 \\
0.7 & 0.684849172830231 & 0.69026481777657 & 0.69071717866237 \\
0.8 & 1.129457607979309 & 1.13870338730698 & 1.13947695538814 \\
0.9 & 1.776974746594038 & 1.79180568789922 & 1.79305066803341 \\
1.0 & 2.693706630766665 & 2.71636750366666 & 2.71828182845904 \\
\hline
\end{tabular}

Table 3: Numerical Solution for Example 2

\begin{tabular}{llll}
\hline$x$ & \multicolumn{2}{c}{ Errors } & Exact solution \\
\cline { 2 - 3 } & 20 terms & 25 terms & \\
\hline 0 & 0 & 0 & 0.5 \\
0.1 & $1.6003 \mathrm{E}-10$ & $9.2050 \mathrm{E}-12$ & 0.499376169438922 \\
0.2 & $6.4013 \mathrm{E}-10$ & $3.6821 \mathrm{E}-11$ & 0.497518595104995 \\
0.4 & $2.5615 \mathrm{E}-9$ & $1.4734 \mathrm{E}-10$ & 0.490290337854601 \\
0.5 & $4.0044 \mathrm{E}-9$ & $2.3034 \mathrm{E}-10$ & 0.485071250072666 \\
0.6 & $5.7714 \mathrm{E}-9$ & $3.3199 \mathrm{E}-10$ & 0.478913142610576 \\
0.7 & $7.8597 \mathrm{E}-9$ & $4.5240 \mathrm{E}-10$ & 0.471929178183009 \\
0.8 & $1.0166 \mathrm{E}-8$ & $5.8914 \mathrm{E}-10$ & 0.464238345442620 \\
0.9 & $1.1408 \mathrm{E}-8$ & $6.8893 \mathrm{E}-10$ & 0.455960752587553 \\
1.0 & 0 & 0 & 0.447213595499958 \\
\hline
\end{tabular}

Table 4: Numerical Solution for Example 3

The exact solution is $y(x)=\downarrow \frac{1}{4+x^{2}}$. Errors obtained by the proposed method and the exact solution are presented in Table 4.

\section{Conclusions}

In this paper, we have presented a novel approach for solving higher order nonlinear SBVP's. Errors obtained by the proposed method and the exact solution are presented in Tables 2- 4. It is observed from the tables that the DTM solu- 
tion is superior than the method in $[5,6]$ and it is very good agreement with the exact solution. The present approach can be applied directly without requiring linearization, discretization or perturbation. Also, it shows a promising tool for solving a class of higher order nonlinear SBVP's.

\section{References}

[1] K. Sharma, Chaitan P. Gupta, Iterative solutions to non-linear fourth order differential equations through multi integral methods, Inter. J. Comput. Math.,28(1989),219-226.

[2] A. R. Yousef Salah ,M. El-Sayed, A. A. Salama, Iterative solutions of singular fourth-order boundary value problems, Indian J. Pure Appl. Math.,32(1)( 2001),9-16.

[3] A.S.V. Ravi Kanth, Y.N. Reddy, Cubic spline for a class of singular twopoint boundary value problems,Appl.Math.Comput.,170(2)(2005),733740.

[4] A. M. Wazwaz, The variational iteration method for solving nonlinear singular boundary value problems arising in various physical models, Commun. Nonlinear Sci.Numer. Simul.,189(2)(2007), 2017-2022.

[5] A.S.V. Ravi Kanth, K. Aruna, Solution of singular two-point boundary value problems using differential transform method, Phys. Lett. A, 372(26)(2008),4671-4673.

[6] Y. Q. Hasan, L. M. Zhu, Solving singular boundary value problems of higher-order ordinary differential equations by modified Adomian decomposition method, Commun. Nonlinear Sci. Numer. Simul., 14(2009),25922596.

[7] Y. Q. Hasan, L. M. Zhu, A note on the use of modified Adomian decomposition method for solving singular boundary value problems of higherorder ordinary differential equations, Commun. Nonlinear Sci. Numer. Simul.,14(2009),3261-3265.

[8] F. Geng, Solving singular second order three-point boundary value problems using reproducing kernel Hilbert space method,Appl. Math. Comput.,215(6)(2009)2095-2102. 
[9] A.S.V. Ravi Kanth, K. Aruna, He's variational iteration method for treating nonlinear singular boundary value problems, Comput. Math. Appl.,60(2010)821-829.

[10] F. Geng, A novel method for solving a class of singularly perturbed boundary value problems based on reproducing kernel method, Appl. Math. Comput., 218(8)(2011),4211-4215.

[11] A. M. Wazwaz. A reliable treatment of singular Emden-Fowler initial value problems and boundary value problems, Appl. Math. Comput., 217(24)(2011),10387-10395.

[12] A.S.V. Ravi Kanth, Cubic spline polynomial for non-linear singular twopoint boundary value problems, Appl. Math. Comput., 218(8)(2011),42114215.

[13] J.K. Zhou, Differential transform and its Applications for Electrical Circuits, Huazhong University Press, Wuhan, China(1986).

[14] C. K. Chen, S. H. Ho, Application of differential transformation to eigen value problems, Appl. Math. Comput., 79(2-3)(1996),173-188.

[15] M. J. Jang, C. L. Chen, Analysis of the response of a strongly nonlinear damped system using a differential transformation technique, Appl. Math. Comput.,88(2-3)(1997),137-151.

[16] C. L. Chen, Y.C. Liu, Solution of Two-point boundary value problems using the differential transformation method, J. Optim. Theory Appl., 99(1)(1998),23-35.

[17] M. J. Jang, C. L. Chen, Y. C. Liy, On solving the initial-value problems using the differential transformation method, Appl. Math. Comput.,115(23) (2000),145-160.

[18] F. Ayaz, Solution of the system of differential equations by differential transform method, Appl. Math. Comput., 147(2) (2004), 547-567.

[19] M. M. Rashidi, The modified differential transform method for solving MHD boundary-layer equations, Comput. Phys. Commun., $\mathbf{1 8 0 ( 1 1 ) ( 2 0 0 9 ) , 2 2 1 0 - 2 2 1 7 . ~}$

[20] A.S.V. Ravi Kanth, K.Aruna, Differential transform method for solving the linear and nonlinear Klein-Gordon equation, Comput. Phys. Commun., 180(5) (2009) 708-711. 
[21] J. Biazar, M. Eslami, Analytic solution for telegraph equation by differential transform method, Phys. Lett. A, 374(29)(2010),2904-2906.

[22] R. Chiba, Applications of differential transform method to thermo elastic problem for disks of variable thickness with temperature-dependent parameters, Inter. J. Thermophysics, 33(2)(2012),363-380. 
